\title{
$\Omega$
}

Sunita Rai, Alina Andreea Ionescu

Sunita Rai, Alina Andreea lonescu

D

Respiratory Medicine, Aneurin Bevan University Health Board, Newport, UK.

\section{A 44-year-old, Caucasian, male nonsmoker with worsening difficulty in breathing and decreased exercise tolerance}

\section{Case report}

A 44-year-old, Caucasian man was referred to the respiratory team for worsening difficulty in breathing and decreased exercise tolerance in September 2015. He had been admitted to the general surgery department in December 2014, and treated for an acute severe pancreatitis complicated by a lesser sac collection, necrotic pancreatitis and gastric outlet obstruction. The treatment was conservative and supportive; following his discharge from hospital, he had repeated re-admissions with symptomatic ascites needing drainage via paracentesis.

$\mathrm{He}$ was referred to the gastroenterologist department for further management and started diuretics (spironolactone and furosemide) to control his ascites. Despite the medical management, his breathlessness continued to worsen.

He had never smoked, consumed large amounts of alcohol well over the maximum recommended weekly intake, had a previous history of systemic hypertension and hypercholesterolaemia, and worked as a tyre fitter. Medication consisted of furosemide, spironolactone, citalopram, a proton pump inhibitor, mirtazapine and slow-release morphine twice a day for ongoing abdominal pain.

On examination in the respiratory clinic, he looked pale and thin, with normal heart sounds and no pedal oedema, but there were signs of a right-sided pleural effusion. The chest radiograph and point-of-care thoracic ultrasound confirmed a free right-sided pleural effusion. The overall World Health Organization (WHO) performance status was 2.

He underwent a therapeutic pleural aspiration (1.5 $\mathrm{L}$ aspirated) that found a "milky" fluid with a $\mathrm{pH}$ of 7.47, protein content of $39 \mathrm{~g} \cdot \mathrm{L}^{-1}$, glucose of $129.7 \mathrm{mg} \cdot \mathrm{dL}^{-1}$, lactate dehydrogenase of $96 \mathrm{U} \cdot \mathrm{L}^{-1}$, triglycerides of $451.7 \mathrm{mg} \cdot \mathrm{dL}^{-1}$ and cholesterol of $69.6 \mathrm{mg} \cdot \mathrm{dL}^{-1}$.

Task 1

Based on this information, what is your diagnosis?
a. Empyema
b. Chylothorax
c. Pseudo-chylothorax
d. Exudative pleural effusion

Cite as: Rai S, lonescu AA. A 44-year-old, Caucasian, male nonsmoker with worsening difficulty in breathing and decreased exercise tolerance. Breathe 2017; 13: 117-122. 


\section{Answer 1}

b. Chylothorax

The diagnostic criteria for chylothorax based on pleural fluid analysis have been previously described [1]. Based on that study, pleural fluid containing $>110 \mathrm{mg} \cdot \mathrm{dL}^{-1}$ triglycerides strongly support the diagnosis of chylous fluid, whereas $<50 \mathrm{mg} \cdot \mathrm{dL}^{-1}$ triglycerides indicate a nonchylous fluid [1]. This criterion continues to be used in combination with a pleural fluid cholesterol level of $<200 \mathrm{mg} \cdot \mathrm{dL}^{-1}$; the presence of chylomicrons supports the diagnosis of chylothorax, particularly in a small number of cases who present with triglyceride levels $<110 \mathrm{mg} \cdot \mathrm{dL}^{-1}$ in the pleural fluid [2, 3].

4 weeks later, a chest and abdomen computed tomography $(C T)$ was performed in view of his persistent shortness of breath (figure 1).

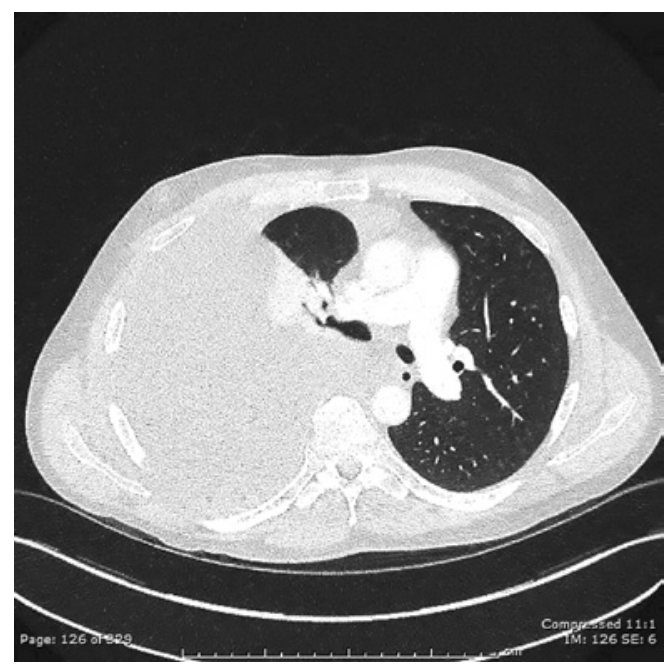

Figure 1 Thoracic CT image.

\section{Task 2}

1 What are the CT findings shown in figure 1?

a. Pleural and pericardial effusion

b. Parapneumonic pleural effusion

c. Large, right-side pleural effusion with displacement of the mediastinum

d. Large pleural effusion and calcified, bilateral pleural plaques

2 What is your next step?

a. Perform repeat therapeutic pleural aspirations

b. Insert an 18 French chest drain

c. Refer to thoracic surgeon for ligation of the thoracic duct

d. Perform pleural drainage and pleural biopsies at thoracoscopy 


\section{Answer 2}

1 c. Large, right-sided pleural effusion displacing the mediastinum to the left. There is very little aerated lung in the right hemithorax.

$2 \mathrm{~d}$. Perform pleural drainage and pleural biopsies at thoracoscopy

He later underwent a video-assisted thoracoscopy (see the video in the supplementary material) under conscious sedation, in view of the unilateral effusion identified on this occasion. The British Thoracic Society guidelines state that in a symptomatic exudative pleural effusion, a thoracoscopy should be performed where there is suspicion of malignancy, or if the pleural aspiration results are inconclusive or negative [4]. Macroscopically, the parietal and visceral pleurae looked inflamed and otherwise normal (no nodular changes); the pleural fluid looked chylous. Biopsies obtained at this time did not show any evidence of malignancy (particularly no signs of lymphoma); the appearances were of a histiocytic and mixed lymphoid infiltrate.

Further to the pleural intervention, the patient's shortness of breath improved but he remained generally frail, his abdominal pain continued to be problematic requiring morphine treatment, and he remained lethargic and cachectic with a WHO performance status of 2 at best.

\section{Task 3}

How would you treat the chylothorax in this case?

a. Refer to thoracic surgeon for thoracic duct ligation

b. Refer back to general surgeon for the management of the abdominal pain

c. Continue best supportive care and involve dieticians for advice

d. No further input is required from the respiratory team 


\section{Answer 3}

c. Continue best supportive care and involve dieticians for advice

The patient was referred to a specialist dietician for a low-fat diet, an essential step to reduce the accumulation of chylothorax. The case was discussed with a thoracic surgeon and a conservative management was recommended.

Gastroenterologists and upper gastrointestinal surgeons continued to monitor his pancreatitis, which slowly improved. Follow-up in the pleural clinic showed a minimal amount of pleural fluid after 2 months of a low-fat diet. His abdominal symptoms slowly improved. However, $\sim 5$ months after being discharged from the respiratory team, the patient was re-admitted to the surgical department with exacerbation of chronic pancreatitis and ascites. He once more had recurrent episodes of ascites requiring drainage. $\sim 6$ weeks later, he had a further admission to the critical care unit with spontaneous bacterial peritonitis and acute kidney injury. Shortly after this, the patient died.

\section{Task 4}

How did the chylothorax most likely occur in this case?

a. Blockage of the thoracic duct by a tumour

b. Injury to the thoracic duct

c. Injury to the cisterna chyli by the pancreatic enzymes

d. Abdominal lymphadenopathy with compression of the thoracic duct 


\section{Answer 4}

c. Injury to the cisterna chyli by the pancreatic enzymes

\section{Discussion}

Chylothorax is the accumulation of chyle (lymphatic fluid of intestinal origin) in the pleural space as a result of disruption or obstruction of the thoracic duct or its branches. Chyle has a milky appearance, and has a high content of triglycerides, chylomicrons and lymphocytes. It is important to distinguish a chylothorax from pseudochylothorax (cholesterol pleurisy), defined as accumulation of cholesterol crystals as a result of chronic pleural effusion. Pseudochylothorax has a similar thick milky appearance but unlike chyle, it does not contain triglycerides or chylomicrons [5]. However, it is important to be aware that not all chylothorax cases present with the classical milky fluid appearance of chyle [2, 3].

Chylothorax is a relatively rare condition that has multiple aetiologies. These include traumatic causes of which surgery, in particular thoracic surgery, is more common than physical injuries such as a vertebral fracture, penetrating trauma or childbirth [2]. The nontraumatic causes include malignancy leading to thoracic duct obstruction (most often lymphoma), amyloidosis, retrosternal goitre, congenital thoracic duct ectasia and sarcoidosis [5, 6].

The case shown developed a rare complication of necrotising pancreatitis. The precise mechanism leading to chylothorax in this condition is debatable. Anatomically, the pancreas is situated in the upper abdomen in the retroperitoneum, close to the duodenum, stomach and liver, as well as to the cisterna chyli (lymphatic sac) formed from the confluence of different lymphatic vessels. This ascends vertically to form the thoracic duct, which passes through the diaphragm, and ascends to empty into the left subclavicular and jugular vein $[2,6]$. Therefore, a possible mechanism is that the activated pancreatic enzymes causing the pancreas to "digest itself" in necrotising pancreatitis could erode the lymphatic vessels and lead to leakage of chyle. Necrotising pancreatitis has a mortality rate between $5 \%$ and $40 \%$ depending on the extent of pancreatic involvement [7]. A chylous ascites can gain access to the pleural space via the diaphragmatic pores, leading to a chylothorax $[6,8]$.

The conservative management of chylothorax involves therapeutic thoracentesis to drain the chyle from the pleural space, in order to allow lung re-expansion and nutritional support. It is advised to keep the patient nil by mouth (for short periods of time) or on a diet rich in low-fat medium-chain triglycerides (MCTs). MCTs reduce the amount of chyle flowing into the thoracic duct by bypassing the intestinal lymphatic system and being directly absorbed into the portal system [9]. This alone may resolve half of the cases of traumatic or congenital chylothoraces [10]. However, if this fails, then total parental nutrition, which decreases the flow of chyle further, may be required, especially in chronic chylothoraces or cases where there is rapid loss of nutrients in the chyle $[9,10]$. As well as malnutrition, there may be electrolyte losses leading to hypocalcaemia or hyponatraemia [11]. Consequently, serum electrolyte levels, total protein and albumin should be monitored, and any deficiencies appropriately corrected [9].

As part of conservative management, somatostatin and octreotide have been used outside routine medical management. These agents reduce intestinal chyle production by inhibiting biliary, pancreatic and gastric secretions, as well as preventing intestinal absorption of fat, mainly triglycerides [12, 13]. They also reduce the rate of lymph flow through the thoracic duct by acting on the somatostatin receptors [12, 13]. As a result, the volume of chyle flowing through the damaged thoracic duct is reduced.

Surgical management is advised after a postsurgical or post-traumatic chylothorax, or if medical management fails (drainage of $>1500 \mathrm{~mL}$ chyle per day in adults). The scope of surgical intervention is to identify the chyle leak and performing thoracic duct ligation, or if the leak cannot be identified, to perform pleural decortication and pleurodesis [14]. In the case we present, due to the patient's frailty, thoracic surgery was considered hazardous.

In conclusion, this case report discusses a rare cause of chylothorax in a young man who was treated for necrotising pancreatitis. The conservative management of his chylothorax allowed him symptomatic relief and a relatively better quality of life before the relapse of his pancreatitis.

\section{Supplementary Material}

This article has supplementary material available at breathe.ersjournals.com

\section{Conflict of interest}

Disclosures can be found alongside this article at breathe.ersjournals.com 


\section{References}

1. Staats BA, Ellefson RD, Budahn LL, et al. The lipoprotein profile of chylous and nonchylous pleural effusions. Mayo Clin Proc 1980; 55: 700-704

2. McGrath EE, Blades Z, Anderson PB. Chylothorax: aetiology, diagnosis and therapeutic options. Respir Med 2010; 104 $1-8$

3. Maldonado F, Hawkins FJ, Daniels CE, et al. Pleural fluid characteristics of chylothorax. Mayo Clin Proc 2009; 84: 129-133.

4. Hooper C, Lee YCG, Maskell N, et al. Investigation of unilateral pleural effusion in adults: British Thoracic Society pleural disease guideline 2010. Thorax 2010; 65: 4-14.

5. Hillerdal G. Chylothorax and pseudochylothorax. Eur Respir J 1997; 10: 1157-1162.

6. Sukumaran KN, Petko M, Hayward MP. Aetiology and management of chylothorax in adults. Eur J Cardiothorac Surg 2007; 32: 362-369.

7. Pal KM, Kasi PM, Tayyeb M, et al. Correlates of morbidity and mortality in severe necrotizing pancreatitis. ISRN Surg 2012; 2012: 215193.
8. Romero S, Martín C, Hernandez L, et al. Chylothorax in cirrhosis of the liver: analysis of its frequency and clinical characteristics. Chest 1998; 114: 154-159.

9. de Beer HG, Mol MJ, Janssen JP. Chylothorax. Neth J Med 2000; 56: 25-31.

10. Fernández Alvarez JR, Kalache KD, Graŭel EL. Management of spontaneous congenital chylothorax: oral medium-chain triglycerides versus total parenteral nutrition. Am J Perinatol 1999; 16: 415-420.

11. Servelle $M$, Noguès $C$, Soulié J, et al. Spontaneous, post-operative and traumatic chylothorax. J Cardiovasc Surg 1980; 21: 475-486.

12. Al-Zubairy SA, Al-Jazairi AS. Octreotide as a therapeutic option for management of chylothorax. Ann Pharmacother 2003; 37: 679-682.

13. Kalomenidis I. Octreotide and chylothorax. Curr Opin Pulm Med 2006; 12: 264-267.

14. Marts BC, Naunheim KS, Fiore AC, et al. Conservative versus surgical management of chylothorax. Am J Surg 1992; 164: 532-535. 